\title{
THE EFFECTS OF REASONING, PRIOR MOOD, AND PERSONALITY ON EMOTION
}

\author{
Mike OAKSFORD ${ }^{1)}$, Juliette CARLILE ${ }^{1)}$ and Simon C. MOORE ${ }^{1)}$ \\ ${ }^{1)}$ Cardiff University, U.K.
}

\begin{abstract}
Whether performing a syllogistic reasoning task (SRT) affects emotional state in different prior moods was tested using eighty participants in either a positive, negative, or neutral mood. A film mood induction procedure (MIP) was used and affective state was assessed before the MIP, after the MIP and after the SRT. The affective reactivity hypothesis - that personality influences the magnitude of affective change to an MIP - was also tested by having participants fill out the Eysenck Personality Inventory. Participants in a positive, but not in a negative or neutral, prior mood moved more negative after the SRT. Participants' pattern of affective reactivity partly replicated previous research but contrary to prediction in the positive condition extraversion was inversely related to increases in positive (in the MIP) and negative (in the SRT) affect. These results suggest that, under some conditions, people may adaptively move to the mood-state that is most conducive to the type of cognitive task they must perform.
\end{abstract}

Key words: reasoning, emotion, extraversion, introversion, affective reactivity

Research into cognition and emotion has typically concentrated on the effects of emotional states on cognitive performance. There has been much less attention paid to how performing different cognitive tasks may affect our emotions. Yet according to recent cognitive theories (Oatley \& Johnson-Laird, 1987, 1995) emotions are elicited by significant cognitive events that may occur in the performance of a task. For example, sadness (or a negative emotional state) may be elicited by the "failure of a major plan or the loss of an active goal" (Oatley \& Jenkins, 1996, p. 256). Such events are likely to occur in the performance of many high level cognitive tasks, such as reasoning or planning. The purpose of the experiment we report here was to assess the effects of performing a reasoning task on people's emotional state. Participants performed two syllogistic reasoning tasks and had their emotional state assessed before and after performing the tasks (we describe the syllogistic reasoning task fully in the Method section).

One possible source of prediction for how different tasks may affect emotional state is to look at the literature on how the emotions affect cognitive task performance. For example, positive affect seems to facilitate creative thinking (e.g., Isen, Daubman \& Nowicki, 1987, but see, Kaufmann \& Vosburg, 1998). If positive affect facilitates creative thinking, then it would be adaptive if creative thinking induced a positive affective state. Similarly, reasoning and planning performance would appear to be at normal levels when in a neutral or negative mood but is suppressed in a positive mood

Correspondence concerning this article should be addressed to Mike Oaksford, School of Psychology, Cardiff University, Tower Building, Park Place, Cardiff, CF10 3AT, Wales, U.K. (oaksford@cardiff.ac.uk). 
(e.g., Oaksford, Morris, Grainger, \& Williams, 1996). Consequently, it might be adaptive if performing a reasoning task when in a positive mood induced a neutral or negative emotional state.

This line of reasoning suggests that a person's mood state prior to performing a cognitive task may influence how their emotional state is affected. There is evidence broadly consistent with this view. It is well known that people in different emotional states may approach a cognitive task in different ways. One classic finding is that people in a positive mood are less willing to wager on a high risk bet than controls (Arkes, Herren, \& Isen, 1988; Isen \& Geva, 1987; Isen \& Patrick, 1987). An interpretation of this effect is that people are risk averse in this situation because they associate positive utility with maintaining a positive affective state (Isen, 1987). Losing a lot of money is likely to make them more negative and so they wager less money than the controls. This result suggests that people in different emotional states may perform differently on a cognitive task because of an awareness of how the task may affect them emotionally. Consequently, in this experiment we induced both a positive and a negative emotional state prior to the reasoning task to assess the effects of prior mood-state. Consistent with the previous literature we predicted that participants in a positive mood would move to a neutral emotional state. However, because systematic or analytic processes like reasoning seem unaffected in either a negative or a neutral mood (Petty \& Cacioppo, 1986; Schwarz $\&$ Bless, 1991), we predicted that the reasoning task should not affect participants' emotional state in these conditions.

In this experiment we also investigated the effects of personality. Some studies have shown a positive relationship between extraversion and positive affect and between neuroticism and negative affect (for recent summaries see, Gross, Sutton \& Ketalaar, 1998). For example, Larsen and Ketelaar $(1989,1991)$ showed that extraversion predicted the magnitude of the change to a more positive mood bought about by a positive mood induction. Moreover, they showed that neuroticism predicted the magnitude of the change to a more negative mood bought about by a negative mood induction. Thus extraverts seem reactive to positive but not to negative mood and neurotics seem reactive to negative but not to positive mood. These personality differences have only been investigated with respect to specific mood induction procedures, e.g., music, films, Velten's self statements, that researchers have used to explicitly alter people's emotional state prior to performing a cognitive task. However, to our knowledge, whether the emotion-altering properties of performing a cognitive task are similarly modulated by personality has not been investigated. In this experiment we investigated this question for a reasoning task.

Previous research on affective reactivity has assumed that people start in the same affective state and has then manipulated mood to investigate how personality variables modulate people's reaction to a mood manipulation (e.g., Gross et al., 1998). In our experiment there was a mood induction phase just as in previous research. However, after this phase we effectively introduced a further mood manipulation, the reasoning task, which was expected to make people feel more negative. This further manipulation allowed us to investigate whether prior mood modulates affective reactivity to a negative mood induction. The previous research on personality and mood reactivity we discussed 
above predicted that there would be a greater change to a negative mood for neurotics in all conditions with no effect of extraversion. However, such a prediction assumes that prior mood or the reasoning task does not modulate mood reactivity. This experiment also investigated whether this assumption is true.

In this experiment participants were put into either a positive or negative mood state using a film mood induction procedure (MIP) and then performed a syllogistic reasoning task (SRT). Their emotional state was then assessed using self-report scales (Isen et al., 1987) before the MIP, after the MIP (and before the SRT), and after the SRT. We included a neutral film control condition and a no-film control condition. After the post SRT affect ratings had been elicited the participants completed the Eysenck Personality Inventory (Eysenck \& Eysenck, 1972). We expected that the reasoning task would induce a negative emotional state and we expected that prior mood would modulate the effects of personality on mood reactivity.

\section{METHOD}

\section{Participants:}

There were 80 participants $(9$ males), all were undergraduate psychology students at the School of Psychology, Cardiff University, and all were volunteers. Participants had a mean age of 19.9 years $(S D=3.5)$ with a range of 18 to 36 years. The gender distribution of this sample was predominantly female reflecting the distribution of Psychology students at Cardiff University.

Design:

The design was a 4 (Mood: positive, negative, neutral, control) $\times 2$ (Personality: high or low, extraversion or neuroticism) quasi-experimental factorial design. Participants were assigned randomly to one of four mood conditions, so that there were 20 participants in each condition. Two of the four conditions involved a mood manipulation - positive and negative - by means of films combined with a self induced mood manipulation (see, Oaksford et al., 1996; Slyker \& McNally, 1991). The remaining conditions consisted of a neutral-film and a no film control. Participants were assigned to a high or a low extraversion or neuroticism group depending on their EPI scores. Each participant performed an abstract and a thematic syllogistic reasoning task in one of the two possible orders. All participants were tested individually.

\section{Materials:}

Film clips. Similar film clips to those used by Oaksford et al. (1996) were used in this experiment. The comedy film clip, used to induce positive affect, was 7 minutes of You've Been Framed, a television program containing a number of video clips sent in by viewers and containing humorous incidents. The negative film clip was 7 minutes of an Amnesty International video "Prisoners of Conscience" and consisted of interviews with political prisoners. Pilot work indicated that such material induced the desired mood state. Isen et al. (1987) used a harrowing film of Nazi concentration camps to induce negative mood. They argued that these materials were likely to induce more extreme mood states than those desired. Consequently a milder negative mood inducement was used in this experiment. The neutral film clip was 7 minutes of the BBC nature program Wild Life on One. In the final control condition participants did not see a film clip.

Reasoning task: The reasoning tasks consisted of an abstract and thematic version of a logically valid syllogism. In the thematic version participants read the following passage and were then asked to pick the conclusion they thought followed from the list (the first two sentences in italics correspond to the premises of the syllogism). The materials were taken from Mayer (1992):

As technology advances and natural petroleum resources are depleted, the securing of petroleum from unconventional sources becomes more imperative. One such source is the Athabasca tar sands of northern Alberta, Canada. Since some tar sands are sources of refinable hydrocarbons, these deposits 
are worthy of commercial investigation. No sources of refinable hydrocarbons are found in kerogen deposits.

Therefore:

All tar sands are kerogen deposits.

No tar sands are kerogen deposits.

Some tar sands are kerogen deposits.

Some tar sands are not kerogen deposits.

None of these conclusions is valid.

The logically correct conclusion is in italics (although it was not in italics in the instructions). The abstract version of the task provided no context and replaced tar sands with A, sources of refinable hydrocarbons with $\mathrm{B}$, and kerogen deposits with $\mathrm{C}$.

Eysenck Personality Inventory. The EPI (Eysenck \& Eysenck, 1972) is a 57 item questionnaire with 24 yes-no questions relating to neuroticism and 24 to extraversion. The remaining questions form part of the liar scale to assess whether people are answering the questions consistently. The extraversion and neuroticism scales are independent and in this sample they were not correlated, $r(78)=.14$. The liar score in this sample had a mean of $2.33(S D=1.5)$ and showed no relationship with either extraversion $(r(78)=.16)$ or neuroticism $(r(78)=.10)$. Participants also showed no differential tendency to lie in the different mood conditions, $F(3,76)<1$.

Procedure:

Participants were tested individually. On entering the experimental room participants were seated at a desk on which the video player and monitor were set up. Participants completed the EPI after the experiment.

In the positive and negative conditions the experimenter then read the following instructions out aloud to the participants (based on Slyker \& McNally, 1991):

"In this experiment you will first be asked to provide some information about your current mood state using some ratings scales that are attached to the desk in front of you. I am then going try to get you to alter your mood state in a particular direction with the help of a film (I am then going to show you a film.) I will ask you to rate your mood state again after the film. Finally I will ask you to perform a brief task. The whole experiment should only take around 25 minutes."

In the neutral film condition the sentence in italics was replaced by the sentence in brackets. In the control condition only the first sentence of this passage was used and was followed by: "I will then ask you to perform a brief task. The whole experiment should only take around 10 minutes."

In the three film conditions participants then completed the before MIP mood check that consisted of five 9-point Likert scales (see, e.g., Isen et al., 1987): refreshed vs. tired; calm vs. anxious; alert vs. unaware; positive vs. negative; and amused vs. sober. Each scale had a positive end (refreshed, calm, alert, positive, amused) and a negative end (tired, anxious, unaware, negative, sober). The lower end of the numerical scale was assigned to positive feelings. The composite score, summing across all items, was used to assess affective state. Other studies have used just the positive-negative scale treating the other items as fillers. However, the more items that are used the more likely these scales are to provide a valid and reliable indicator of affective state (Kidder \& Judd, 1986). These mood manipulation checks were carried out before the MIP, after the MIP (and so before the SRT) and after the SRT. This allowed the construction of change scores for the MIP and the SRT. These scores were used to assess the affective reactivity hypothesis.

In the positive mood condition, on completing the mood manipulation check participants were told:

"What I would now like you to do is to try and get into a good or happy (bad or unhappy) mood. The film I will show you is designed to help you get into that mood. You can also use images, memories, or any other way you can think of to help you get into the mood. The film will last around 7 minutes, and then I will ask you to rate your mood again. You should try hard to get into a good (bad) mood. But also, be honest when you rate your mood later. So, if your mood doesn't change, your ratings should show that. Any questions."

(In the negative condition the items in brackets replaced the items in italics; in the neutral condition these instructions were not read out.)

Participants were then shown the appropriate film clip. When the film was over they were asked to fill in the after-MIP mood manipulation check. In the control condition participants went directly from filling in a single mood manipulation check to the reasoning task. 
Participants were given the abstract and thematic syllogistic reasoning tasks in the order to which they had been assigned. In both tasks they were instructed to pick the conclusion they could be sure of. Once they had completed both reasoning tasks participants filled out the final, post SRT mood manipulation check. Finally they completed the EPI. They were then thanked for participating and debriefed about the purpose of the study.

\section{RESUlTS AND DISCUSSION}

\section{Mood Manipulation Checks}

Table 1 shows the results of the mood manipulation checks. We averaged over the 5 items to produce mean pre and post MIP affect ratings. Within film conditions comparisons showed that after seeing the positive film participants were more positive than before they saw the film, $t(19)=10.68, p<.0001$, that after seeing the negative film participants were more negative than before they saw the film, $t(19)=5.10, p<.0001$, but that after seeing the neutral film there was no significant change in participants' emotional state, $t(19)=1.83, n s$. For between film comparisons we collapsed the post MIP affect ratings for the neutral and control conditions because the neutral film led to no changes in affective state. We then performed a between subjects ANOVA with the post MIP affect ratings as the dependent variable. Participants in the positive condition were significantly more positive and participants in the negative condition were significantly more negative than those in the neutral/control condition (mean $=4.40, S D=1.17$ ) both at the .005 level in post hoc Scheffe tests. These results showed that the film MIPs successfully produced increases in the target emotions.

We also compared the before MIP scores to check whether participants were equated for emotional state before receiving the mood manipulations. The overall ANOVA was significant, $F(3,76)=2.93, M S E=.96, p=.039$. Post-hoc Tukey HSD tests revealed only one significant difference: participants were already more negative in the negative film condition than in the neutral film condition at the .05 level. Finding that participants are not all equated for affective state prior to a mood induction is common in this type of research. However, as we found that it was the negative group that was more negative and that they went more negative as a result of the film MIP, this result could not question any interpretation attributing differential effects to the mood factor. The lack of any other significant differences also showed that the neutral condition did not differ from the control condition in the pre MIP scores. This result further motivates collapsing these conditions (see above).

\section{Personality and Affective Reactivity: The MIP}

The MIP provides the opportunity to test the affective reactivity hypothesis that we discussed in the introduction, that extraverts are more reactive to positive mood and neurotics are more reactive to negative mood. This hypothesis has typically been assessed using regression analysis. This analysis strategy implies a model where a personality factor like extraversion varies continuously with affective reactivity. However, other models are possible. For example, rather than varying continuously in the population, 
Table 1. Mean Affect Ratings and Change Scores in Each Mood Condition

\begin{tabular}{lccccc}
\hline \multirow{2}{*}{ Condition } & $\begin{array}{c}\text { Pre MIP Mean } \\
\text { Affect Rating }\end{array}$ & $\begin{array}{c}\text { Post MIP Mean } \\
\text { Affect Rating }\end{array}$ & $\begin{array}{c}\text { MIP Change } \\
\text { Score }\end{array}$ & $\begin{array}{c}\text { Post SRT Mean } \\
\text { Affect Rating }\end{array}$ & $\begin{array}{c}\text { SRT Change } \\
\text { Score }\end{array}$ \\
\cline { 2 - 6 } & $M(S D)$ & $M(S D)$ & $M(S D)$ & $M(S D)$ & $M(S D)$ \\
\hline Positive & $4.71(.70)$ & $3.40(.78)$ & $1.31(.55)$ & $4.69(.94)$ & $-1.29(1.11)$ \\
Negative & $5.21(1.19)$ & $5.74(1.06)$ & $-.53(.47)$ & $5.74(1.13)$ & $0(.52)$ \\
Neutral & $4.30(1.03)$ & $3.97(1.24)$ & $.33(.81)$ & $4.35(1.16)$ & $-.38(.61)$ \\
Control & 0 & $4.82(.93)$ & 0 & $4.90(1.13)$ & $-.08(.71)$ \\
\hline
\end{tabular}

Note: The control condition shows zeros because no post-mood induction test was possible and so change scores could not be computed for this condition. MIP: Mood Induction Procedure, SRT: Syllogistic Reasoning Task.

there may be a threshold. People whose extraversion levels are above this threshold may on average have higher levels of affective reactivity. However, for the subgroups above and below this threshold there may be no relationship, or at least no consistent linear relationship, between the two constructs. This may be because factors other than extraversion also influence affective reactivity and these factors may be different above and below the threshold. In this situation it would be theoretically possible for significant differences in affective reactivity to be detected between sub and supra threshold participants although no significant correlation was observed between extraversion and affective reactivity. Because of this possibility, we also analysed our data factorially by dividing participants into high and low extraversion and neuroticism groups using a median split of the overall data. Splitting by extraversion the high group had a mean score of $15.70(S D=1.95)$ and the low group had a mean score of $9.73(S D=2.75)$. Splitting by neuroticism the high group had a mean score of $14.60(S D=2.80)$ and the low group had a mean score of $7.20(S D=2.31)$. The median split was carried out across mood conditions. ${ }^{1}$ Nonetheless, within mood conditions there were still almost equal numbers of participants in the high and low groups.

To assess the mood reactivity hypothesis the most straightforward approach is to regress the MIP change scores on the extraversion scale. However, as Gross et al. (1998) observe, sometimes the pre MIP affect ratings correlate with the change scores. For this reason they used a residualised change score. The post MIP affect ratings were regressed on the pre MIP affect ratings and the residual was then used as the dependent variable in further regression analyses. This procedure means that the relationship between affective reactivity and personality was assessed holding pre MIP affective state constant. In our

\footnotetext{
${ }^{1}$ Because of the number of ties for the median value we moved the smallest number of ties into the high or low group. For example, if the median value was 12 and the 40th, 41st and 42nd participants all had scores of 12 , then we would move the 40th up into the high group, now defined as having a score $\geq 12$. Alternatively, if the 39 th, 40th and 41 st participants all had scores of 12 , then we would move the 41 st down into the low group, now defined as having a score $<12$.
} 
Table 2. Correlations Between Personality and Affective Reactivity for the MIP Change Scores and Residualised Change Scores

\begin{tabular}{cccccc}
\hline \multirow{2}{*}{ MIP } & \multicolumn{2}{c}{ Neuroticism } & & \multicolumn{2}{c}{ Extraversion } \\
\cline { 2 - 3 } \cline { 5 - 6 } & Change Score & Resid. Score & & Change Score & Resid. Score \\
\hline Positive & -.33 & -.34 & & $-.62^{*}$ & $-.61^{*}$ \\
Negative & .05 & .12 & & .29 & .10 \\
Neutral & -.05 & -.06 & & -.34 & -.33 \\
\hline
\end{tabular}

*=Significant at at least the .05 level. Resid. Score: Residualised Change Score.

$N=20$ in each condition.

data the pre MIP affect ratings correlated with the change scores for the negative condition. We therefore computed the correlations using both the MIP change scores and the residualised change scores for all conditions. The correlations are shown in Table 2, where it can be seen that there was only any disagreement between these two measures for the negative condition.

Table 2 reveals an unexpected pattern of affective change with personality. The affective reactivity view predicts that neuroticism should correlate with increases in negative emotion and that extraversion should correlate with increases in positive emotion. However, in our results there was no relationship between neuroticism and affective change in the negative condition. Moreover, in the positive condition there was an inverse correlation between extraversion and affective change. All participants in the positive condition showed an increase in positive affect. However, higher increases were associated with lower levels of extraversion. This was exactly opposite to prediction. Moreover the correlation had a large effect size (-.62) in comparison to Gross et al. (1998) who observed only a medium sized effect (.24) in the opposite direction. One might argue that Gross et al.'s results are more reliable because of the larger sample size $(N=156$, compared to $N=20)$. However, given the effect sizes observed, the experiments were equivalent in power (post-hoc analysis): Gross et al. (1998), power $(1-\beta)=.92$, our experiment, power $=.96$.

This finding was further confirmed in two $2 \times 4$ ANOVAs with either extraversion (high, low) or neuroticism (high, low) and film condition as factors with the MIP change score as the dependent variable. There were no significant effects for neuroticism. However, for extraversion, although neither main effect nor interaction was significant, in simple effects comparisons the change to a positive mood for the positive group was lower for the high extraverts $(1.07(.57))$ than for the low extraverts $(1.60(.37)), F(1,54)=3.72$, $M S E=.37, p=.059$. No other simple effect approached significance.

There would appear to be two possible explanations for this effect. First, it could be due to the gender distribution in our sample: $88.75 \%$ of our participants were female compared to $47.44 \%$ in Gross et al. (1998). There are two reasons to dismiss this explanation. First, if women predominantly show a large inverse correlation then how did 
Gross et al. observe a positive correlation in a sample with an even split of males and females? Even if the positive correlation occurred predominantly for men, one would have expected the effects to cancel. Moreover, Gross et al. (1998) made no mention of any gender differences. Second, to anticipate our results, the predominantly female participants in our experiment did reveal some of the predicted affective reactions in response to the SRT.

The second possible explanation concerns the self-induced mood manipulation (Slyker \& McNally, 1991) we used in this experiment where participants were explicitly asked to try to get into a positive or negative mood. It is possible that explicit instruction to change affective state may interact with personality to alter the relation with the magnitude of affective change. This could happen if introverts were generally more compliant and extraverts were generally more rebellious and less willing to take instruction. However, such an account does not explain the lack of an effect for neuroticism. Clearly this is an area that requires further investigation. However, it is an important finding that how personality influences affective reactivity may depend on whether the mood manipulation is implicit or explicit. ${ }^{2}$

\section{Effects of Reasoning on Affective State}

In the Introduction we argued that research on how affective states influence reasoning tasks (Oaksford et al., 1996) suggested that performing the reasoning task may induce a negative affective state, in particular for participants in a positive mood. To test this prediction we performed a $2 \times 4$ mixed ANOVA, with film-condition as a between subjects factor, with mood before and after as two levels of a within subjects factor and with the affect ratings as the dependent variable (see Table 1 for means). Consistent with prediction participants were significantly more negative after performing the reasoning task, $F(1,76)=12.60, M S E=1.94, p<.00001$. Consequently it would seem that performing an analytic reasoning task has a profound effect on emotion moving people to a more negative affective state.

There was also a significant main effect of film condition, $F(3,76)=25.85, M S E=.30$, $p<.00001$, and a significant interaction, $F(3,76)=11.81, M S E=.30, p<.00001$. We further investigated the interaction using simple effects comparisons. Consistent with hypothesis, participants were significantly more negative after performing the reasoning task in the positive condition, $F(1,76)=56.18, M S E=.30, p<.00001$, but not in the control, $F(3$, $76)<1$, or in the negative conditions, $F(3,76)<1$. Participants in the neutral film condition also showed a smaller move to a negative $\operatorname{mood}$ state, $F(3,76)=4.88, M S E=.30, p<.05$. This would appear to be because on average these participants moved towards a positive mood as a result of the MIP. Table 1 shows that those groups (positive and neutral) who moved to a positive mood as a result of the MIP have moved back to their pre MIP affect levels as a result of the SRT. Participants in the negative condition revealed no change to a more negative state after the SRT.

\footnotetext{
${ }^{2}$ We also assessed whether there were any relations between personality and levels of affect (Gross et al., 1998). This meant correlating the individual affect ratings with extraversion and introversion. There were no significant correlations for any of the three affect ratings taken during this experiment.
} 
Table 3. Mean Proportion Correct $(S D)$ by Extraversion (High-Low) and by Film Condition

\begin{tabular}{ccccc}
\hline \multirow{2}{*}{ Extraversion } & Positive & Negative & Neutral & Control \\
\cline { 2 - 5 } & $M(S D)$ & $M(S D)$ & $M(S D)$ & $M(S D)$ \\
\hline High & $.23(.34)$ & $.27(.34)$ & $.27(.41)$ & $.55(.37)$ \\
& $N=9$ & $N=12$ & $N=12$ & $N=12$ \\
Low & $.44(.46)$ & $.44(.39)$ & $.39(.49)$ & $.75(.35)$ \\
& $N=11$ & $N=8$ & $N=8$ & $N=8$ \\
\hline
\end{tabular}

A possible explanation for this pattern of affective change is that people are equally able to engage in analytic reasoning in either a neutral or a negative mood (Petty \& Cacioppo, 1986; Schwarz \& Bless, 1991). Consequently if participants are already in a negative mood there is no reason for their affective state to move more negative to permit efficient reasoning performance. Moreover, for participants in a positive mood there is no reason for their affective state to move more negative than is required to allow efficient reasoning. This explanation assumes that affective state can adaptively change to facilitate the varying cognitive tasks that we must engage in as we go about our daily lives. If we are right then we might expect that performance on the SRT should be at similar levels in all mood conditions. Moreover in all mood conditions we should find unimpaired levels of performance, i.e., they should be at similar levels as found in other research on syllogistic reasoning.

In a meta-analysis of syllogistic reasoning research carried out by Chater and Oaksford (1999) the mean percentage correct for the syllogism used in this experiment was $44.3 \%$. In the current experiment this percentage was $41.25 \%$. That is, performance was at comparable levels. There were also no differences between the mood induction conditions, as predicted. However, overall there were close to significant differences in percentage correct responding between the high $(32.56 \%$ [SD=37.61]) and the low extraverts $(51.35 \%[S D=43.28]), F(1,72)=3.95, M S E=.16, p=.051$ (see Table 3). Consistent with previous research (see, Koelega, 1992, for a summary) it would appear that the high extraverts were poorer at sustaining attention in the reasoning task than the low extraverts. We also found that the control group's performance, at a mean of $65 \%$ $(S D=36.64)$ correct, was much better than the norms reported in Chater and Oaksford (1999). We offer no explanation for this effect. The results of the reasoning task are consistent with findings showing that although positive mood suppresses analytic task performance, a negative or neutral mood allows normal levels of performance (Oaksford et al., 1996; Petty \& Cacioppo, 1986; Schwarz \& Bless, 1991). After the SRT most participants in this experiment were either in a negative or a neutral affective state.

\section{Affective Reactivity: The SRT}

Table 4 shows the correlations between personality and the SRT change scores and the residualised SRT change scores in each mood condition. The results show a pattern of 
Table 4. Correlations Between Personality and Affective Reactivity for the SRT Change Scores and Residualised Scores

\begin{tabular}{lccccc}
\hline \multirow{2}{*}{ MIP } & \multicolumn{2}{c}{ Neuroticism } & & \multicolumn{2}{c}{ Extraversion } \\
\cline { 2 - 3 } \cline { 5 - 6 } & Change Score & Resid. Score & & Change Score & Resid. Score \\
\hline Positive $(N=20)$ & .21 & .11 & & $.51^{*}$ & $.40^{*}$ \\
Negative $(N=20)$ & .17 & .21 & & .22 & .18 \\
Neutral $(N=20)$ & .20 & .27 & & .17 & .12 \\
Control $(N=20)$ & -.18 & -.20 & & .21 & .23 \\
All-Pos. $(N=60)$ & .15 & $.24^{*}$ & & .20 & .17 \\
All $(N=80)$ & .02 & .12 & & $.27^{*}$ & $.23^{*}$ \\
\hline
\end{tabular}

*=Significant at at least the .05 level (one-tailed). Resid. Score: Residualised Change Score.

results partly consistent with previous research on affective reactivity and partly inconsistent with that work. On the one hand when we collapsed across the negative, neutral and control conditions there was a positive relation between neuroticism and increases in negative emotion using the residualised change scores. This finding replicates Gross et al. (1998). However, some caution is required in interpreting this result. Gross et al. used the residualised change score because it was reasonable to assume that differences in pre MIP mood state were due to differences in using the scales. Consequently it was reasonable to use the residualised scores which treat participants as equated for emotional state before the MIP. However, in this experiment we explicitly changed people's mood prior to the SRT, and so there is no reason to assume they were equated for mood before the reasoning task. Nonetheless, as we show below, this result also came out in factorial analyses of the same data using the standard change scores.

For the positive condition extraversion was inversely correlated with increases in negative affect. ${ }^{3}$ So the lower the level of extraversion the greater the change to a negative affective state. Although the number of participants was smaller the power of the present experiment was close to that of Gross et al.'s (1998) experiment: standard change score, $1-\beta=.80$; residualised change score, $1-\beta=.59$. Therefore we can be confident that this is quite a robust result. It implies that changes to a negative affective state are modulated by prior mood. Although, when in a negative or neutral affective state, increases in negative affect are positively correlated with neuroticism, when in a positive affective state, increases in negative affect are inversely correlated with extraversion. This latter relationship also held in the overall data (see Table 4, final row).

We also investigated these data factorially. In a $2 \times 4$ ANOVA with neuroticism and film-condition as between subjects factors and the SRT change score as the dependent variable, we found no significant main effects or interactions. However, excluding the

\footnotetext{
${ }^{3}$ The sign of the correlations is positive because the sign of the change scores (Post MIP Affect Rating - Post SRT Affect Rating) were negative indicating a change to a negative mood.
} 
Table 5. Mean SRT Change Scores by Extraversion (Hi-Lo) and by Film Condition

\begin{tabular}{ccccc}
\hline \multirow{2}{*}{ Extroversion } & Positive & Negative & Neutral & Control \\
\cline { 2 - 5 } & $M(S D)$ & $M(S D)$ & $M(S D)$ & $M(S D)$ \\
\hline High & $-.73(.62)$ & $.11(.38)$ & $-.31(.52)$ & $.04(.91)$ \\
Low & $-1.98(1.21)$ & $-.13(.64)$ & $-.47(.73)$ & $-.20(.45)$ \\
\hline
\end{tabular}

positive condition did reveal a main effect of neuroticism, $F(1,54)=4.87, M S E=.37$, $p<.05$. High neurotics $(.29[S D=.69])$ showed a greater increase in negative affect than low neurotics $(.06[S D=.47]))$. This result is consistent with the regression analysis and with Larsen and Ketelaar (1989, 1991).

A similar analysis but using extraversion instead of neuroticism as a between subjects factor revealed significant main effects of extraversion, $F(1,72)=8.73, M S E=.51$, $p<.005$, and film condition, $F(3,72)=14.94, M S E=.51, p<.00001$, with a close to significant interaction, $F(3,72)=2.64, M S E=.51, p=.056$. The relevant means are shown in Table 4 (affect ratings are shown as negative when the change was towards a negative mood). We further investigated the interaction using simple effects comparisons. These comparisons revealed that the change to a more negative mood state was significantly greater for the low-extraverts only in the positive film condition, $F(1,72)=15.24$, $M S E=.51, p<.0001$ (in the remaining three conditions, $F(1,72)<1$ ). These analyses were consistent with the regression analyses of affective reactivity.

These results suggest that how personality affects mood reactivity to negative events may depend on the mood that someone is in. Consistent with previous research (Larsen \& Ketelaar, 1989, 1991; Gross et al., 1998), in a neutral or negative mood, increases in negative affect are correlated with neuroticism. However, in a positive mood, increases in negative affect are inversely correlated with extraversion. It is possible that the latter finding was due to the low extraverts in the positive condition also being high neurotics. This would predict a significant difference in reactivity between the low and the high neurotics in the positive condition, which we did not observe. More weakly, perhaps extraversion and neuroticism were anti-correlated in the positive condition, which again might partially explain this result. However, if anything there was a positive correlation between extraversion and neuroticism, albeit not significant, $r(18)=.26, p=.27$. Therefore in a positive mood it would appear that affective reactivity to negative events depends on extraversion, not on neuroticism.

\section{CONCLUSIONS}

This experiment has produced two interesting findings. First, and perhaps most importantly, it would appear that if someone is in a positive affective state then an analytic cognitive task that requires systematic cognitive processing will move them to a neutral or 
negative affective state. This would appear to be an adaptive response because previous work has shown that people's systematic reasoning may be impaired in a positive mood but not in a neutral or negative mood (e.g., Bless \& Schwarz, 1999; Oaksford et al., 1996; Petty \& Cacioppo, 1986; Schwarz \& Bless, 1991). However, it does raise some puzzling questions. In particular, if people's affective state adaptively changes to the state most conducive to performing a cognitive task, then why in countless experiments are performance differences observed between people in different induced mood states? Surely at the onset of the cognitive task people's affective state should go to that most conducive to performing the task and so no performance variation should be observed. However, in many experiments affective state is not assessed after the cognitive task so it is simply not known whether it has changed as a result of performing the task. Moreover, a whole range of other factors must influence whether such an adaptive change will come about. These factors will include, for example, the strength of the initial MIP, the duration and nature of the cognitive task, the time course of the change in affective state bought about by the onset of the cognitive task, the motivation of participants to engage in the cognitive task, personality variables like need-for-cognition and so on. Consequently, our conclusion must be stated more cautiously: people's affective state may adaptively change to that most conducive to performing the task, for some tasks, under some conditions. We now know that syllogistic reasoning is one such task. However, what those conditions are and why they were satisfied in this experiment requires further research.

The second finding of interest was that the patterns of mood reactivity we observed seemed to depend on two unexpected factors. First, the pattern of affective reactivity to the SRT seemed to depend on prior mood state. Consistent with previous research, in a negative or neutral affective state, increases in negative emotion were correlated with neuroticism but were unaffected by extraversion. However, in a positive affective state increases in negative emotion were inversely correlated with extraversion but were unaffected by neuroticism. Second, the pattern of affective reactivity to the MIP differed from previous research where neuroticism has been found to be correlated with increases in negative mood and extraversion has been found to be correlated with increases in positive mood (Gross et al., 1998; Larsen \& Ketelaar, 1989, 1991). In our experiment neuroticism was not correlated with increases in negative mood and extraversion was inversely correlated with increases in positive mood. We argued that this different pattern of affective reactivity may be due to the explicit or self induced nature of our mood induction procedure. This is an area clearly in need of further investigation.

What are the mechanisms that could cause the effects we have observed? We have argued that the SRT induced change to a negative mood in the positive condition is consistent with accounts that identify negative mood with analytic processing and positive mood with heuristic processing. The standard explanation of this phenomenon (but see, Bless \& Schwarz, 1999) is that positive mood leads to either decreased processing motivation or decreased processing resources (Schwarz \& Bless, 1991). Either is hypothesised to lead to shallower more heuristic processing of information. Our results seem broadly consistent with either view. One could argue that because these participants were highly motivated the switch to the new goal has led to increased processing 
motivation. Alternatively one could argue that for the same reason, participants immediately devote more resources to the task. However, without further research into the conditions that brought about this apparently adaptive change in mood it is too early to speculate on which account might be correct.

A final cautionary note that seems to follow from these results is that if the performance of cognitive tasks substantially affects people's mood states, then we can never be sure that they perform the task in the emotional state induced in the MIP. To guarantee this requires mood checks post cognitive task performance and preferably during the performance of the task. This may require the use of more continuous and objective methods of measuring emotions rather than the punctate self-report measures used in this and in many other experiments.

\section{REFERENCES}

Arkes, H. R., Herren, L. T., \& Isen, A. M. 1988. The role of potential loss in the influence of affect on risktaking behaviour. Organisational Behaviour and Human Decision Processes, 42, 181-193.

Bless, H., \& Schwarz, N. 1999. Sufficient and necessary conditions in dual-mode models: The case of mood and information processing. In S. Chaiken \& Y. Trope (Eds.), Dual-process theories in social psychology (pp. 423-440). New York, NY: The Guilford Press.

Chater, N., \& Oaksford, M. 1999. The probability heuristics model of syllogistic reasoning. Cognitive Psychology, 38, 191-258.

Eysenck, H. J., \& Eysenck, S. B. G. 1972. Manual of the Eysenck personality questionnaire. San Diego, CA: Educational and Industrial Testing Service.

Gross, J. J., Sutton, S. K., \& Ketelaar, T. 1998. Relations between affect and personality: Support for the affect-level and affective-reactivity views. Personality and Social Psychology Bulletin, 24, 279-288.

Isen, A. M. 1987. Positive affect, cognitive processes and social behaviour. In L. Berkowitz (Ed.), Advances in experimental social psychology (Vol. 20, pp. 203-253). New York: Academic Press.

Isen, A. M., Daubman, K. A., \& Nowicki, G. P. 1987. Positive affect facilitates creative problem solving. Journal of Personality and Social Psychology, 52, 1122-1131.

Isen, A. M., \& Geva, N. 1987. The influence of positive affect on acceptable risk: The person with a large canoe has a large worry. Organisational Behaviour and Human Decision Processes, 39, 145-154.

Isen, A. M., \& Patrick, R. 1987. The effect of positive feelings on risk taking: When the chips are down. Organisational Behaviour and Human Decision Processes, 31, 194-202.

Kaufmann, G., \& Vosburg, S. K. 1997. "Paradoxical” mood effects on creative problem-solving. Cognition and Emotion, 11, 151-170.

Kidder, L. H., \& Judd, C. M. 1986. Research methods in social relations (5th ed.). New York: Holt, Rinehart and Winston.

Koelega, H. S. 1992. Extroversion and vigilance performance -30 years of inconsistencies. Psychological Bulletin, 112, 239-258.

Larsen, R. J., \& Ketelaar, T. 1989. Extroversion, neuroticism, and susceptibility to positive and negative mood induction procedures. Personality and Individual Differences, 10, 1221-1228.

Larsen, R. J., \& Ketelaar, T. 1991. Personality and susceptibility to positive and negative emotional states. Journal of Personality and Social Psychology, 61, 132-140.

Mayer, R. E. 1992. Thinking, problem solving, cognition. San Francisco: W. H. Freeman.

Oaksford, M., Morris, F., Grainger, B., \& Williams, J. M. G. 1996. Mood, reasoning and central executive processes. Journal of Experimental Psychology: Learning, Memory and Cognition, 22, 476-492.

Oatley, K., \& Jenkins, J. M. 1996. Understanding emotions. Oxford: Blackwell.

Oatley, K., \& Johnson-Laird, P. N. 1987. Towards a cognitive theory of emotions. Cognition and Emotion, 1, 29-50. 
Oatley, K., \& Johnson-Laird, P. N. 1995. The communicative theory of emotions: Empirical tests, mental models and implications for social interaction. In L. L. Martin \& A. Tesser (Eds.), Goals and affect. Hillsdale, NJ: Erlbaum.

Petty, R. E., \& Cacioppo, J. 1986. The elaboration likelihood model of persuasion. In L. Berkowitz (Ed.), Advances in experimental social psychology (Vol. 19, pp. 124-205). New York: Academic Press.

Schwarz, N., \& Bless, H. 1991. Happy and mindless, but sad and smart? The impact of affective states on analytic reasoning. In J. P. Forgas, (Ed.), Emotion and social judgments. International series in experimental social psychology (pp. 55-71). Oxford, England UK: Pergamon Press.

Slyker, J. P., \& McNally, R. J. 1991. Experimental induction of anxious and depressed moods: Are Velten and musical procedures necessary? Cognitive Therapy and Research, 15, 33-45.

(Manuscript received April 1, 2004; Revision accepted October 22, 2004) 